Working in the Methodological 'Outfield': the Case of Bourdieu and Occupational Therapy

Jo Watson $^{\text {a }}$ and Michael Grenfell ${ }^{\mathrm{b}}$

${ }^{a}$ Faculty of Health Sciences, University of Southampton, UK.

${ }^{b}$ School of Education, University of Stirling, UK

Corresponding author: Dr Jo Watson, Faculty of Health Sciences, University of

Southampton, Highfield, Southampton, Hampshire SO171BJ, UK. jdw@soton.ac.uk

Word Count : 6877 


\title{
Working in the Methodological 'Outfield': the Case of Bourdieu and Occupational Therapy
}

The article reports on a study of methodological innovation involving Occupational Therapy (OT) students in higher education. It is based on an original project which examined the experiences and outcomes of non-traditional entrants to pre-registration OT education.

\begin{abstract}
A feature of the original project was the application of the epistemological and methodological approach of the French social theorist Pierre Bourdieu, most noticeably in exploring the way that social back ground (habitus) interacted with the educational (field) context in terms of experience and educational outcome.
\end{abstract}

Bourdieu used a ranged of techniques - both qualitative and quantitative - in collecting and analysing data. In particular, he used Multiple Correspondence Analysis (MCA), a type of geometric data analysis recognised as a powerful tool enabling the representation of social space and the situating of individuals within it with respect to a number of variables.

The article considers methodological principles in comparing ethnographic, traditional statistics and MCA. We show how the original data were re-analysed according to MCA. The article compares the original analyses and findings with those based on MCA in order to explore its strength over the previous approach and the potential it has to cast light on various issues in higher education.

Keywords: Bourdieu; methodology; multiple correspondence analysis, higher educational research; occupational therapy 


\section{Working in the Methodological 'Outfield': the Case of Bourdieu and Occupational Therapy}

\section{Introduction}

This article reports on a BERA sponsored project to explore and extend the applications of the methodological approach envisioned by the French social theorist Pierre Bourdieu. Bourdieu became known by the education constituency after the publication of seminal texts in the 1970s (1971a, 1971b) which, together with such a British counterpart as Basil Bernstein (see 1975, 1996), established him as an educational sociologist of international repute with further agenda setting texts (see Bourdieu 1993, 1988, 1996). Bourdieu wrote extensively outside of an educational research context, although many of his associated works on language, culture and politics are also pertinent to it. Common to all his work is a commitment to develop what he called a 'metanoia' (1992, 251), or 'new gaze', on social phenomena. This new perspective was driven through methodological concerns which addressed issues of theory and practice in new and innovative ways.

Bourdieu is now an influential figure with many researchers from a diverse academic field drawing on his ideas, including those from education where he has continued to inform a range of enquiries. A key issue for Bourdieu was research methodology; his own work taking on board a number of qualitative and quantitative techniques in new and original ways. In education, empirical investigations undertaken from a Bourdieusian perspective have hitherto tended to adopt a qualitative approach; this also being the dominant methodology of educational research in the UK, at least in recent decades. However, Bourdieu also used statistics 
extensively in his own work, and from the earliest studies. Later, he adopted Multiple Correspondence Analysis (MCA) as, in his words,

'it is a relational technique of data analysis whose philosophy corresponds exactly to what, in my view, the reality of the social world is. It is a technique which 'thinks' in terms of relation, as I try to do precisely in terms of field' (Bourdieu and Wacquant 1992, 96).

At the core of this article is a pre-existing study of occupational therapy students which was undertaken from a Bourdieusian perspective using both ethnographic and statistical methods. Our original principal aim in this current BERAsponsored project was to reanalyse the data using MCA: firstly, to train ourselves in a statistic method which is both sophisticated and obviously accords with Bourdieu's social theoretical vision; and secondly, to examine what this affords us in terms of additional insights into the original data. In other words, what does MCA show that the original ethnographic study and statistics did not? - and indeed, vice versa - and thus what methodological scope and framework would be pertinent for other studies in educational research. The article is framed to contribute to the debate around mixed methods in social science research; in particularity of numerical and non-numerical data.

There is a second pertinent methodological point important to note here. Bourdieu was, and is, primarily known as a sociologist; and thus by implications is mostly interested in questions of class, power, gender, race and the like. However, it is important to draw a distinction between his own so-called sociology and what he called an ‘Anglo-saxon’ version. Without going into all the detail of this 
differentiation, we would make three key points. Firstly, Bourdieu was originally trained as a philosopher, and much of his own epistemology is underpinned by close readings in continental philosophy (for example, Husserl and Heidegger), which is not necessarily so in sociology in an English speaking world. Moreover, his own education was heavily influenced by French thinking; in particular phenomenologists such a Merleau-Ponty, and historians of the philosophy of science such as Bachelard and Canguilhem. Finally, Bourdieu's approach is heavily infused with moral philosophy and anthropology; indeed, one is as likely to read about ethnology as sociology in his own writing.

We see these as critical issues both in framing our article and indeed the empirical and methodological practice underpinning it. We understand this essential sociological/anthropological approach to the social world has relevance in broader contexts outside the normal preoccupations of sociology. Our own study originates in research into the pre-registration education of occupational therapists. We were interested in this group as a constituency - who they were, where they came from, their age, gender and ethnic profiles as indicators of responses and reactions in the processes of becoming a qualified therapist. These concerns are broadly sociological, but we would wish to emphasise that our key intent is to better understand them as processes, not just to see provenance-outcome findings as an end in themselves. We are further concerned with exploring how MCA might sharpen this understanding as compared with the conventional approaches adopted in the original study.

The article is divided into three further main sections. After elucidation of principles and issues with respect to the qualitative/quantitative divide and Bourdieu 
in the next section, we present the original project, its research questions and its findings. We then give an account of the MCA application and what this showed. The discussion section compares the original with the MCA study and considers the advantages and disadvantages of both. The final concluding section then brings together a number of methodological and theoretical points by way of evaluating the potential of Bourdieu and MCA in particular for future educational research studies.

\section{Background}

Bourdieu famously wrote that in the social sciences nothing was more 'fundamental and ruinous' than the opposition between subjectivism and objectivism' (1990, 25). And, of course, by implication we might extend the statement to 'theory and practice' and 'qualitative and quantitative' approaches to research, all of which are implicated with each other. It is commonly accepted that whilst ‘qualitative methods’ pertain more to individual, subjective, interpretative knowledge, ‘quantitative techniques’ can be viewed more nomothetically; that is, establishing 'objective’ facts and patterns. Of course, the divide is not so clear cut: the qualitative does aspire to facts which are objective - up to a point - whilst the quantitative always involves a degree of individual, subjective, interpretation. It is therefore also commonly accepted that the best approach to research employs both: quantitative to give scope and breadth and qualitative to give depth. Indeed, this is exactly what Bourdieu did in early studies on Algeria (Bourdieu 1958, 1962, 1963, 1964) and his own home Béarn territory (2008). However, issues existing here are not just about methodological etiquette but relate to the conditions of knowledge formation itself, and thus the status of what is known. 
Bourdieu's own work was nearly always developed in the field, and the practical requirements needed there to understand what was going on. He writes of how key concepts such as field, habitus and cultural capital were 'logically necessitated' by the empirical facts, by submersion in the data, whether qualitative or quantitative. Clearly, for Bourdieu, the 'empirical subject' needed to be distinguished from the 'scientific' one $(1995,7)$, all whilst recognising that the latter was prone to 'radical doubt’ (Bourdieu 1992, 235ff; Grenfell 2004, 199); indeed, that this was a hallmark of good research, not a weakness. Naïve interpretation could never be a valued end in itself, but a stage along the way to forming 'science'. At the same time, he warns against ‘crushing one’s rivals' with so-called 'objective' statistics (1999a, 10) and, clearly, for him, the Popperian notion of 'knowledge without a knowing subject' would be anathema. What we find in his work is a range of approaches, both qualitative and quantitative, to meet the needs of the job in hand and balanced accordingly; from the population studies of early research in Algeria (1958), to the questionnaire surveys of education and museum (1990b), from the intricate MCA analyses of the academic field $(1988,1996)$, to the later intimate interviews portraying the 'weight of the French world' (1999). All of these methods and approaches are based on one fundamental concern for Bourdieu: structure.

We might say that the basis of Bourdieu's science is the simple fact of a coincidence between an individual's connection with both the material and the social world, which thus needs to be understood in terms of structure. Everything lies in this structural connection, in the links that are established between human beings and phenomena - the structures of primary sense, feeling and thought - the intensional (sic. See Bourdieu 1968; Grenfell 2012, 43-47; Grenfell and Lebaron 2014, 11-12), 
material and ideational- with which they come in contact. This is a basic phenomenological precept. The 's' here is intended to draw attention to this structural relationship.

Such an understanding is also central to research activity. We can say that everything about Bourdieu's methodology is about structures: in the construction of the research object, in the levels of field analysis, and even in participant objectivation (see Grenfell 2012, chapter 13). Structure is, therefore, at the base of Bourdieu's science, and attempts to transcend the opposition between subjectivity and objectivity. Ultimately, this dichotomy is also played out methodologically in terms of a further distinction: one substantialist, the other relational. The substantialist approach treats things as pre-existing entities, with essential properties - as realist objects, whilst the relationalist approach understands things in terms of their relational context - how they acquire sense in terms of their position with respect to other phenomena which share their context (see Bourdieu 1998, 3-6). So, when Bourdieu uses a term such as dispositions, he is intending them not as actual, hidden entities, but as existing only in as much as they are part and parcel of social and psychological structures in their mutually constituting existence. The real is relational: what exists in the social world are relations - not interactions between agents or intersubjective ties between individuals, but objective relations which exist independently of individual consciousness and will (Bourdieu 1989). Bourdieu makes a distinction between the actual structure of the social system in its multidimensional stratification, and the symbolic products which arise from it: 'In reality, the space of symbolic stances and the space of social positions are two independent, but homologous, spaces' (Bourdieu 1994/87, 113). This is an attempt to reconstruct the space of differences, or 
differential positions to be found in social space. It is then possible to account for these positions as differential properties of habitus, dispositions, capital, etc.

In our project, we have taken the results from one study and reworked the data from an MCA perspective. MCA allows us to reconstruct the structure of the sub-field of occupational therapy education and to identify what is symbolically significant as dispositional capital in terms of the topography of student recruitment and outcome.

\section{The original project}

Students from less privileged social backgrounds continue to be under-represented in UK higher education (HE). Examples of successful participation are naturally evident, but the overall pattern of trajectories of this group of students differs notably from that of traditional entrants to HE (see Reay 2006). The implicit expectation in the HE field is that students will adapt to its established practices and culture, which generally remains oriented towards traditional white middle-class populations (Burke 2005).

Spanning the fields of $\mathrm{HE}$ and professional practice, pre-registration occupational therapy (OT) as a sub-field in education in the UK is influenced by policies in both arenas aiming to increase diversity. Mature students exceed $60 \%$ of the national intake (COT 2010) and, although ethnic minorities and men are underrepresented, many students now enter from more diverse backgrounds. A substantial number have prior experience of working in the health and social care sector (Craik 2006) and anecdotally many enter with 'non-traditional' academic backgrounds. 
A three-year longitudinal case study centring on a pre-registration OT programme in one of the UK's research intensive universities sought to answer the key research question: How do OT students from non-traditional academic backgrounds negotiate the learning requirements of the HE environment? Thirteen volunteer participants were recruited from a single cohort and data were collected from them through initial focus groups exploring pre-entry educational experiences and expectations of studying in HE, reflective diaries contemporaneously recording educational experiences in the field that each participant considered significant or meaningful, and one-to-one semi-structured interviews conducted towards the end of participants' first and third years of study further exploring their learning experiences in HE. Background demographic information was collected and each participant generated a ‘family education and employment map’. Analysis of documentation underpinning educational delivery (such as institutional, school and/or departmental mission statements, strategy and policy documents, regulations, validation documents and programme specifications, timetables, module profiles and assessment criteria) provided insight into the pervading culture of the field context - the values and assumptions of the case study site (Bogdan and Biklen 2007). Depth and further contextual information to aid the understanding of student experiences was provided by examining the progression routes of 239 students registered on the same programme in relation to their age at entry, gender, entry qualifications, ethnicity and socio-economic background and exit awards (including sub-honours awards).

Bourdieu's theory of practice provided a framework for the analysis of qualitative data and the development of a new conceptual model identifying academic, linguistic, social and professionally-oriented capital as underpinning the 
logic of practice of this microcosm of the HE field (see Watson 2013a for a detailed account). The culture and practice of the sub-field entered was identified as having a marked influence on participants' experiences within it and on the way that they navigated the demands made of them. For example, those whose habitus was most closely aligned to the pervading culture of the field held the strongest initial portfolios of capital (based on, for example, the status of their academic qualifications, their linguistic repertoire and the strength and nature of their social networks) and therefore occupied more comfortable positions within the field in terms of appreciating and responding appropriately to the practices and expectations encountered. The extent to which participants' positions within the field were enhanced, static or undermined during their engagement with it reflected their ability to develop 'a feel for the game' (Bourdieu 1990a, 66) and where necessary adapt their habitus, and their ability to accrue or extend portfolios of the identified forms of capital. With capital profitable in terms of its practical consequences rather than as inert products of the field (see Grenfell and James 1998), participants who secured a legitimate position within the field, however marginal, were able to covert these portfolios into higher-value cultural capital in the form of the academic/professional qualification which would grant them entry to the OT profession.

Although this might seem an unconventional area in which to deploy Bourdieu's theory as the research question did not focus specifically on social background, the data provided rich illustrations of the influence of academic, linguistic, social and professionally-oriented capital on the experiences and trajectories of participants, spanning those who were very much 'fish in water' 
(Bourdieu and Wacquant, 1992, 127) to those marginalised by or excluded from the field.

The quantitative element of the original research focused on an analysis of the progression routes and exit awards of OT students with non-traditional academic backgrounds compared to those with traditional A-level entry qualifications (see Watson 2013b for a full account). To help set the scene for the discussions that follows, the sample comprised 239 students from four cohorts studying at the case study institution and had the following characteristics:

- mean age at entry was 24.4 years

- nearly $48 \%$ were mature students aged 21 years or older at entry

- women comprised $89 \%$ of the sample (reflecting the typical gender ratios of the profession)

- nearly 95\% self-classified as White British or Irish

- $42.3 \%$ were from National Statistics Socio-economic Classifications (SEC) groups 1 and 2 (higher groups), while 20.9\% were from SEC groups 5-7 (lower groups with a work history).

While academic award is a blunt outcome measure of development and achievement in HE, academic progress and attainment do contribute to the overall picture and add to the context within which student experiences might be understood. Therefore, following appropriate management of the raw data to avoid violating inherent assumptions in this type of analysis (Field 2009, 274), binary logistic regressions were used to analyse the progression routes data based on the successive outcomes of passing at National Qualifications Framework (NQF) Levels 4, 5 and 6 
and the achievement of a 'good' (upper second or first class) honour degree (see Table 1 below). These finding will be used to compare and contrast the insights provide by this more traditional statistical approach and those of MCA.

\section{TABLE 1 POSITIONED APPROXIMATELY HERE}

While data on ethnicity was excluded as it was heavily biased towards 'White British’ and could not be meaningfully collapsed, academic background, maturity at entry and a background from among the middle socio-economic groups had no statistically significant influence on outcome in any of the four analytical models reported on in Table 1. However, there was a consistent pattern of male gender and backgrounds from amongst the lower socio-economic groups acting as significant predictors of poorer outcomes at each level of analysis, with each having a significant influence even when the effect of the other was held constant. As identified by the statistically significant p-values marked by asterisks in Table 1, male gender was the stronger predictor of a poor outcome in relation to passing at NQF Level 4, while a less privileged socio-economic background was a significant predictor of poorer outcomes regarding passing at NQF Level 5 and 6 and securing a good honours degree. In this way, binary logistic regression analysis of OT students’ progression routes provided new insights into the limits of efforts to diversify the profession and raised important questions about the extent to which a discipline which values, respects and supports diversity in the populations it serves is doing so in the education of its own future workforce. 


\section{Extending the analysis using MCA}

Binary logistic regressions offer a means of determining whether successive categorical outcomes can be predicted on the basis of categorical or continuous predictor variables and subsequently ranking the relative importance of meaningful predictors (Field 2009). The results also offer a degree of comparison within each meaningful predictor in that they can be expressed as odds ratios. For example, with reference to Table 1, the odds of a male student failing to secure a good degree are over three and a half times higher than for a female, and the odds of a student from the lower socio-economic groups failing to secure a good honours degree are more than three times higher than for a student from a higher socio-economic group. However, they essentially rank influential variables and do not consider them in relation to each other or as part of a 'bigger', more complex social picture.

Rather than the ‘sociology of variables’ offered by more traditional statistical approaches (La Roux and Rouanet 2010, 11), including binary logistic regressions, MCA can provide a relational alternative that is more sympathetic to an analysis of social fields using a Bourdieusian framework, and indeed as we have already noted, was his preferred approach. With a paucity of applications in educational research, MCA was therefore used to re-analyse the progression routes data described in the previous section as a methodological intervention and extension of the original work to explore if and how this approach might add to the findings.

MCA is best suited to analysing the data emerging from questionnaires based on categorical answer options in which a single response is selected for each question and each response category is associated with only a single question. The original 
research was not framed, nor was data collected, with MCA in mind so the corpus of progression routes data was re-coded appropriately and incomplete datasets were excluded, resulting in 235 cases which were analysed using Coheris SPAD 7.4 ( software. Entry qualifications (comprising 12 categorical response options) and exit awards (with eight categories) were identified as the active variables, while age at entry (eight categories), gender (two categories), ethnicity (eight categories) and SEC (eight categories) were identified as supplementary variables. From a statistical perspective, working with only two active 'questions' (as they would have been had a questionnaire approach originally been utilised) and 18 active categories was not problematic, although from a sociological perspective the constructed space will inevitably be limited (Bonnet 2012 per comm). There are clearly issues regarding the size of the sample, the number of variables and categories and therefore the subsets of the data. This is, however, an illustrative methodological exploration and not an indepth sociological analysis in itself. A richer dataset incorporating responses to a wider and more varied range of carefully selected questions would provide much greater variation in the pattern of selected answers across individual cases and therefore a more nuanced and robust perspective of the social forces in action within the field. Nevertheless, the data enabled us to achieve a primary goal of the project being reported upon - training ourselves in the application of MCA.

The following sections will describe the outputs of the reanalysis using MCA, the interpretation of those outputs and how they compare to the findings of the original binary logistic regressions. 


\section{Examining the cloud of categories}

MCA techniques produce so-called 'clouds of categories' and 'clouds of individuals', each constructed around axes and defined by a table of principle coordinates. For the purposes of this article, which is to exemplify the potential of MCA, we will be focussing on investigating the first two axes which define the plane the best fits the clouds (further details of the axes, eigenvalues and rates of variance can be found at Appendix 1). While the small number of active 'questions' and the small response sub-sets within them necessarily limit its explanatory power, Figure 1 (below) illustrates the cloud of categories produced during analysis of the progression routes data which identifies key response categories from the two active questions, and will form the basis for further discussion regarding the contribution of MCA to expanding understanding of the data. The size of each of the categorical symbols in Figure 1 provides an indication of the proportion of the cases analysed falling into each category (see Appendix 2). The more often individual cases reflect selection of the same answer options for discrete 'questions' (in this case, variables), the closer together those categories will be positioned in the cloud of categories. For example, with reference to Figure 1, A-level qualifications and upper second class honours degrees are positioned in close proximity towards the lower left-hand quadrant of the cloud of categories, indicating that those with A-level entry qualifications were much more likely to achieve an upper second class exit award than, for example, those with Access entry qualifications, who were positioned much further towards the upper left quadrant of the cloud. The overall aim of analysis of the cloud of categories is to determine the similarities between all elements on one side of the origin of each axis and contrasting or opposing them with all that is similar between elements on the other side. 


\section{FIGURE 1 POSITIONED APPROXIMATELY HERE}

In our figure, the cloud is not particularly dense because of limitations within the data. However, this is a positive advantage here since it allows us to focus on subtle but significant variable variation between individuals in uncovering the social forces at play. In Figure 1, for example, we see that mature A-levels and graduate entry qualifications, both of which, by definition, are associated with mature entrants, are positioned to the extreme right of Axis 1. Opposing them on the other side of the axis are a range of 'non-traditional' entry qualifications. Together with graduate and mature A-Level entry qualifications, Access entry qualifications are noteworthy because all exceed the average, and therefore make strong, contributions to the structure of Axis 1 (see Appendix 3 for further information about specific contributions).

The ten cases recording graduate entry qualifications were excluded from the original regression analysis as previous successful engagement with HE was considered likely to influence progression and achievement in subsequent undergraduate awards. The close proximity on Axis 1 of this category to the award of first class honours degrees in the cloud or spread of categories would seem to support this assumption. Binary logistic regressions indicated that the nature of academic background had no statistically significant influence on outcomes, but close scrutiny of the cloud of categories offers a rather more nuanced perspective. 'Non-traditional' entry qualifications are not universally clustered in proximity to each other; sharp oppositions are clearly evident between mature A-Levels and Access qualifications. While acknowledging that the specific subject areas studied in both qualifications 
could potentially vary, entry requirements demand that they are related and relevant to the programme studied in higher education. The cloud of categories represented in Figure 1 therefore highlights an important distinction between how these two 'nontraditional' entry qualifications might influence the position of individuals within the social field being examined. Although these two qualifications are sufficient to grant entry to this particular sub-field of HE, there is clearly a distinction between the value of the capital they afford within it and therefore the position of individuals within the social space.

First class honours degrees also made an above-average contribution to the structure of Axis 1 of the cloud of categories and are positioned to its extreme right, although all other active exit awards (represented in Figure 1 by squares) are positioned to the left of the origin. Taking a composite view of the response patterns evident on Axis 1, we see that it opposes at its extremes Access entry qualification profiles on the left with First class honours profiles on the right, particularly those of graduate entrants and students with mature A-level entry qualifications who achieve first class honours degrees. The implications of this observation are that there is something particular about those holding Access qualifications, if not the qualifications themselves, which marks those students out as having a very different profile from those achieving first class degrees. In Bourdieu's terms, although graduate entrants, those with A-levels achieved as mature students and those with Access entry qualifications are all mature entrants to the field, it would appear that they bring with them very different portfolios of capital which have a marked impact on their positioning with the field. The location of these categories within the cloud represented in Figure 1 draws very clear distinctions between these participants in the 
social space, and highlights the implications for the value of the capital that they are likely to accrue as a result. A richer dataset involving a wider range of answer responses would provide greater stability to the dataset and the opportunity to explore this distinction in more depth but, as will become apparent in the next section, it is still possible to interrogate the available data further.

Focusing now on the vertical axis of the cloud of categories in Figure 1, Access, (traditional) A-level and graduate entry qualifications exceed the average and therefore made strong contributions to the structure of Axis 2, as did the absence of any exit award at all, which is positioned in the extreme upper left quadrant (see Appendix 3). Axis 2 highlights an opposition between (traditional) A-level entry qualification profiles below the origin of the axis and profiles that fail to secure any exit award at all, particularly the profiles of graduates and those with Access entry qualifications who fail to secure an award, above the origin. The distance between the 'no award' and 'graduate' categories in Figure 1 is indicative the fact that only two graduates failed to secure an exit award. The closer proximity between 'no award' and 'Access' suggests that these two variables were much more likely to coincide. The cloud of categories is therefore highlighting again that the capital value within the field of an Access entry qualification is markedly different to that afforded by traditional A-level entry qualifications. It is the latter qualification towards which the established practice and culture of the HE field are historically oriented, so Figure 1 provides an illustration of the challenges potentially encountered by non-traditional entrants, the struggles occurring within the field and the tendency for reproduction of the logic of practice. 
While the cloud of categories provides a useful initial framing of MCA analysis, it is the differences between individuals that offer the greatest insight into a social space. In MCA, this is represented by the form and shape of the cloud of individuals, and the more comprehensive the dataset collected, the more nuanced the picture that emerges. The following section will examine and interpret the cloud of individuals produced for the progression routes dataset that we have been examining, and draw comparisons with the findings of the original binary logistic regressions analysis of the same data.

\section{Examining the cloud of individuals}

Figure 2 below illustrates the cloud of individuals produced for the progression routes data, highlighting that there were 24 different composite response patterns displayed across the 235 cases, with an individual case identified to illustrate each. The relatively small number of composite response patterns is symptomatic of the limitations of the progression routes dataset previously discussed; nevertheless, the cloud of individuals offers valuable additional insights. The more 'average' an individual case is in terms of the responses provided across the range of questions, the closer to the centre of the cloud it will be positioned. Conversely, individual cases located towards the periphery of the cloud are so positioned on the basis of more unusual combined answer patterns. This enables the researcher to discern much more about the social world in terms of the influence of individuals' characteristics than is ever possible using more conventional statistical analysis.

For example, individual 187 at the extreme right of Axis 1 in Figure 2 was a young mature (aged 21-25 years) female student from an 'intermediate occupations', white 
British background who entered with A-level qualifications achieved as a mature student and exited with a first class honours degree. Also on the extreme right, individual 179 was a young mature female graduate from a professional, white British background who also exited with a first class honours degree. Individual 151 on the extreme left was a young mature male student who declined to provide other details about his background, entered with an Access qualification and left with a lower second class honours degree. Similarly, individual 235 was a male who entered with an Access qualification, although he was an older mature student (aged 41-45 years) on this occasion; he was from a white British, semi-routine SEC background and exited without having gained any academic award at all. We therefore begin to see an opposition between white British women from middle to higher SEC groups who entered as mature students and exited with first class honours degrees on the right of Axis 1, and mature male entrants with Access qualifications who exited with a 'lesser' quality honours degrees at best on the left. This analysis reflects the overall findings of the binary logistic regressions, which highlighted that men and those from lessprivileged social backgrounds were less likely to achieve a 'good' honours degree.

\section{FIGURE 2 POSITIONED APPROXIMATELY HERE}

Moving beyond this, however, the cloud of individuals presents an opportunity to further investigate the influence of the supplementary variables such as gender, age at entry and SEC, through the identification of sub-clouds summarised by concentration ellipses providing geometric representations of a structuring factor (La Roux and Rouanet 2010, 69-71). The following sub-sections will interrogate further the cloud of individuals through examination of some key concentration ellipses. 
Whilst again acknowledging possible limitations of the dataset size that small changes in response profiles might impact on the outcome of the analysis, we argue that the resultant ellipses are indeed indicative of underlying structural features dispersed across the field, and can be corroborated by the qualitative findings of the original study.

Age at entry concentration ellipses

The concentration ellipses for age at entry are provided in Figure 3, which also identifies the number of 'responses' to each age-band answer options'. This was the clearest example of sub-clouds produced in the analysis, so will serve well as an initial illustrative exemplar for the purposes in this article. There are no ellipses for the 46-50 or 51-55 age groups as the numbers in these groups were so small. Each ellipse is a geometric representation of the concentration of individual points comprising that sub-cloud around its mean (represented by the position of the marker for the name of the sub-cloud). While there is no clear separation of ellipses in Figure 3 , it is immediately apparent that the mean point of the young, 18-20 years of age school-leavers sub-cloud is on the opposite side of the origin of Axis 2 compared to the mean points of the other five mature entrant sub-clouds, highlighting the structuring effect of age at entry. The deviation between the mean point of the 18-20 group (-0.56) and the 41-45 group (0.85) along Axis 2 is 1.41 which, having wellexceeded La Roux and Rouanet's $(2010,59)$ base-line criterion of 1.0, is indicative of a large effect. The deviation between the 18-20 group (-0.56) and the 36-40 group (0.45) is 1.01 and therefore also indicates a large effect. 
While binary logistic regressions indicated that age at entry had no statistically significant influence on progression through the programme or the achievement of a good honours degree, reanalysis of the data using MCA provides a different perspective. Close analysis of the cloud of individuals identified the existence of a large effect size between the 18-20 sub-cloud and (a) the 41-45 sub-cloud (the mean of which is furthest from the 18-20 mean) and (b) the 36-40 sub-cloud (the mean of which is closest to the 18-20 mean). It is therefore reasonable to conclude that not only did age at entry have a large effect on the structure of the cloud of individuals, opposing young school leavers and mature entrants, but that it is a characteristic that interacts with the social forces operating in the field in very distinct ways. While binary logistic regressions gave no indication of the impact of age at entry, MCA identifies it as having a significant effect on the positioning of an individual within the social field.

\section{Gender concentration ellipses}

Inferential statistics undertaken in the original research found that the final degree mark achieved by women $(\mathrm{M}=63.44$, SE 0.32$)$ was only marginally higher than that achieved by men $(\mathrm{M}=62.49, \mathrm{SE}=1.26)$ and that the difference was not statistically significant $(t(193)=.83, \mathrm{p}=.41)$. However, as highlighted in Table 1 , further scrutiny of the data using binary logistic regressions demonstrated that male gender was a statistically significant predictor of poorer outcomes regarding passing at NQF Levels 4, 5 and 6 and securing a good honours degree. Figure 4 provides the concentration ellipses for gender overlying the cloud of individuals produced by MCA. It is immediately clear that not only is the mean point for the Female sub-cloud positioned on the opposite side of the origin of Axis 1 compared to that of the Male sub-cloud, it 
is also on the opposite side of the origin of Axis 2. At 0.25, the deviation along Axis 1 between the mean points of the Female (0.03) and Male (-0.22) sub-clouds is not remarkable; along Axis 2, however, the deviation is $-0.08+0.66=0.74$ and is considered notable (see La Roux and Rouanet 2010, 59). MCA therefore concurs with the binary logistic regressions in identifying gender as important factor operating within and structuring the social field.

\section{FIGURE 4 POSITIONED APPROXIMATELY HERE}

As noted when discussing the cloud of categories, Axis 2 highlights an opposition between (traditional) A-level entry qualifications profiles below the origin and profiles that fail to secure any exit award at all above it. We can add to this picture a notable deviation between the mean-point of the Female sub-cloud below the origin of Axis 2 and the mean-point of the Male sub-cloud above it. Again, whilst acknowledging possible limitations as stated above, the emerging analysis is consistent with the findings of the original binary logistic regressions which identified that the odds of a male student failing to secure a 'good' degree are over three and a half times higher than for a female. What MCA adds is a much clearer representation of the relationship between gender, entry qualifications and exit awards in the structural oppositions evident within the field. It highlights a social space within which there is a tendency for male gender, Access qualifications and sub-honours exit awards at best to be positioned differentially from female gender, traditional A-level entry qualifications and upper second class honours. It would again suggest that the logic of practice of this particular sub-field of HE privileges the traditionally dominant 
group - in the case of occupational therapy pre-registration education, women from traditional academic backgrounds.

\section{SEC concentration ellipses}

An important finding of the original binary logistic regressions was that the odds of a student from the lower socio-economic groups failing to secure a 'good' honours degree are more than three times higher than for a student from a higher socioeconomic group. The suggestion was, therefore, that the logic of practice of this subfield of HE favoured those from more privileged social backgrounds - the traditional entrants to the HE sector as a whole. Scrutiny of the MCA concentration ellipses for SEC is again consistent with these findings. Although it is not feasible to include a further figure representing these ellipses, we can report that along Axis 1, there was a notably, and very nearly large, deviation between Routine and Higher Managerial/Professional SECs $(-0.57+0.42=0.99)$. Importantly, however, there was also a large deviation between Routine and Small Employers SECs $(-0.57+0.66=$ 1.23). Although there are similarities in the findings of analysis of the data with respect to SEC using binary logistic regressions and MCA, the latter provides a deeper understanding and highlights that it would be mistaken to imagine that SEC provides a simple or straight-forward structuring effect in the field. Although it does have a an impact on the position of individuals within the social space, it does so in combination with other characteristics and features which combine to provide a more or less profitable portfolio of capital which individuals bring to and develop within this particular field. 
Having focussed in on detailed analysis of key variables delineated by concentration ellipses, we now represent the cloud of individuals as a whole to develop a coherent analytical picture (see Figure 5 below). In the upper left quadrant of the cloud of individuals we see mature students who entered with Access qualifications and exited with a sub-honours award at best (i.e. they may have failed to secure any exit award at all), which represents one end of the extreme of exit awards. In the upper right quadrant of the cloud of individuals we see mature students who entered as graduates or with Mature A-levels (the 'more traditional' entry options available to mature students) and exited with first class honours degrees, representing the other end of the extreme of exit awards. In the lower left quadrant of the cloud of individuals we see school-leavers, those who entered with less traditional entry qualifications (e.g. International Baccalaureates or Open University Science Foundations representing more academic alternatives, and HND, GNVQ, AVCE, BTEC and Foundation degrees representing more vocational alternatives) and those who secured more mid-range exit awards (2:1 or 2:2). Finally, while there is limited activity in the lower right quadrant, we do see school-leavers who entered with Alevels and secured mid-range exit awards (2:1 or 2:2).

\section{FIGURE 5 POSITIONED APPROXIMATELY HERE}

\section{In Conclusion}

As noted at the outset, our main objective in undertaking this exploration and extension of method was two-fold: firstly, to further develop a Bourdieusian approach to this area of research; and secondly, to see if MCA would reveal nuances of difference in our data-set. Furthermore, we were looking to take Bourdieu with us 
into a study of the processes of education and learning; to uncover the subtle forces that are at play and influence both formative and summative outcomes. We believe this project has realised these ambitions. We wanted 'less but more', and the results we describe show how relatively small datasets can provide us with nuances in understanding how it is, in this case, that students respond differentially to training to become occupational therapists. Clearly, a similar approach could now be adopted for other professional groups, naturally based on a larger and more robust dataset.

We have also noted how, when Bourdieu is used in educational research, a broadly qualitative and/or ethnographic approach tends to be adopted. These studies do provide important insights. Nevertheless, too frequently, in our view, such approaches lead to a weak form of constructivism, where anything can be shown to influence process. Yet, paradoxically, when MCA is adopted, the technicality of the method results in cloud diagrams which are often allowed to 'speak for themselves'. A further major conclusion from our research project is, therefore, the reaffirmation that both qualitative and quantitative research techniques need to be used together, and that MCA itself, useful though it is, requires analysis complemented by 'natural' data to make the most of its potential. The social world, as Bourdieu recognised, is multi-dimensional and one cannot capture the totality of its complexity. Nevertheless, armed with careful ethnographic insight and Multiple Correspondence Analysis, it is indeed possible to develop a truer picture of the nature of its dynamic and the interaction of its constituent parts. 


\section{REFERENCES}

Bernstein, B. 1975. Class, Codes and Control, Volume III. London: Routledge \& Kegan Paul.

Bernstein, B. 1996. Pedagogy, Symbolic Control and Identity: Theory, Research, Critique. London: Taylor \& Francis.

Bogdan, R. and S. Biklen. 2007. Qualitative Research for Education: An Introduction to Theory and Methods. 5th ed. Boston: Pearson.

Bourdieu, P. 1958. Sociologie de l'Algérie. (New Revised and Corrected Edition, 1961). Paris: Que Sais-je.

Bourdieu, P. 1962. The Algerians (trans. A.C.M. Ross). Boston: Beacon Press.

Bourdieu, P. (with A. Darbel, J.P. Rivet, and C. Seibel). 1963. Travail et travailleurs en Algérie. Paris-The Hague: Mouton.

Bourdieu, P. (with A. Sayad). 1964. Le Déracinement, la crise de l'agriculture tradionelle en Algérie. Paris: Les Editions de Minuit.

Bourdieu, P. 1968. "Structuralism and Theory of Sociological Knowledge", Social Research 35 (4): 681-706.

Bourdieu, P. 1971a/67. "Systems of Education and Systems of Thought.” In Knowledge and Control: New Directions for the Sociology of Education, edited by M.F.D. Young. London: Macmillan.

- “Systèmes d'enseignement et systèmes de pensée.” In Revue Internationale des Sciences Sociales. XIX, 3, 338-88.

Bourdieu, P. 1971b "Intellectual Field and Creative Project.” In Knowledge and Control: New Directions for the Sociology of Education, edited by M.F.D. Young. London: Macmillan.

- “Champ intellectuel et projet créateur.” Les Temps Modernes. Nov: 865-906.

Bourdieu, P. (with J-C. Passeron). 1977/70. Reproduction in Education, Society and Culture (trans. R. Nice). London: Sage.

- La Reproduction. Eléments pour une théorie du système d'enseignement. Paris: Editions de Minuit.

Bourdieu, P. 1988/84. Homo Academicus (trans. P. Collier). Oxford: Polity. - Homo Academicus. Paris: Les Editions de Minuit.

Bourdieu, P. (with L. Wacquant). 1989. "Towards a Reflexive Sociology: A Workshop with Pierre Bourdieu.” Sociological Theory 7 (1): 26-63.

Bourdieu, P. 1990a/1980. The Logic of Practice (trans. R. Nice). Oxford: Polity. - Le sens pratique. Paris: Les Editions de Minuit. 
Bourdieu, P. (with A. Darbel, and D. Schnapper). 1990b/1966. The Love of Art. European Art Museums and their Public (trans. C. Beattie and N. Merriman). Oxford: Polity Press.

Bourdieu, P., and L. Wacquant. 1992. An Invitation to Reflexive Sociology. Cambridge: Polity Press.

Bourdieu, P. 1993. The Field of Cultural Production: Essays on Art and Literature. Oxford: Polity Press.

Bourdieu, P. 1994/ 1987. In Other Words: Essays Towards a Reflexive Sociology (trans. M. Adamson). Oxford: Polity.

Bourdieu, P. (with M. Grenfell). 1995. “Entretiens.” CLE Papers 37. University of Southampton.

Bourdieu, P. 1996/1989. The State Nobility. Elite Schools in the Field of Power (trans. L.C. Clough). Oxford: Polity Press.

- La noblesse d'état. Grandes écoles et esprit de corps. Paris: Les Editions de Minuit.

Bourdieu, P. 1998/1994. Practical Reason. Oxford: Polity Press

- Raisons pratiques. Sur la théorie de l'action. Paris: Seuil

Bourdieu, P. 1999a "Statistics and Sociology." (trans. D. Robbins). UEL Social Politics Paper 10. University of East London.

Bourdieu, P. 1999b/1993. The Weight of the World. Social Suffering in Contemporary Society (trans. P. Parkhurst Ferguson, S. Emanuel, J. Johnson, and S.T. Waryn). Oxford: Polity Press.

Bourdieu, P. 2008. The Bachelors’ Ball: Oxford: Policy

- Le bal des célibataires. Crise de la société en Béarn. Paris: Seuil.

Burke, P. 2005. “Access and Widening Participation.” British Journal of Sociology in Education 26 (4): 555-62.

Craik, C. 2006. “More Graduates - Opportunity or Threat?” Occupational Therapy News 14 (1): 22.

Field, A. 2009. Discovering Statistics Using SPSS. London: Sage.

Grenfell, M. 2004. Pierre Bourdieu: Agent Provocateur. London: Continuum.

Grenfell, M. ed. 2012. Pierre Bourdieu: Key Concepts. Stockfield: Acumen.

Grenfell, M., and D. James. 1998. Bourdieu and Education: Acts of Practical Theory. Abingdon: RoutledgeFalmer.

Grenfell, M., and F. Lebaron, eds.. 2014. Bourdieu and Data Analysis. Berne: Lang. 
Le Roux, B., and H. Rouanet. 1998. "Interpreting Axes in Multiple Correspondence Analysis: Method of the Contributions of Points and Deviations.” In Visualization of Categorical Data, edited by J. Balsius, and M. Greenacre. San Diego: Academic Press.

Le Roux, B. and H. Rouanet. 2010. Multiple Correspondence Analysis. Quantitative Applications in the Social Sciences Series No163. Thousand Oaks: Sage.

Reay, D. 2006. “The Zombie Stalking English Schools: Social Class and Educational Inequality.” British Journal of Educational Studies 54 (3): 288-397.

Watson, J. 2013a. "Profitable Portfolios: Capital that Counts in Higher Education.” British Journal of Sociology of Education 34 (3): 412-430. [published online: 13 August 2012]

Watson, J. 2013b. "Progression Routes and Attainment in Occupational Therapy Education: The impact of Background Characteristics.” British Journal of Occupational Therapy 76 (12): 520-527. 


\begin{tabular}{|c|c|c|c|c|c|c|}
\hline Axis & Eigenvalue & $\begin{array}{c}\text { Rate of } \\
\text { Variance }\end{array}$ & $\begin{array}{c}\text { Cumulated } \\
\text { Rate of } \\
\text { Variance }\end{array}$ & $\begin{array}{c}\text { Modified } \\
\text { eigenvalues }\end{array}$ & $\begin{array}{c}\text { Modified } \\
\text { Rates of } \\
\text { Variance }\end{array}$ & $\begin{array}{c}\text { Cumulated } \\
\text { Modified } \\
\text { Rates of } \\
\text { Variance }\end{array}$ \\
\hline 1 & 0.6437 & $16.05 \%$ & 16.05 & 0.275560604 & 13.69913578 & $13.70 \%$ \\
\hline 2 & 0.5838 & $14.56 \%$ & 30.61 & 0.198066536 & 9.846619315 & $23.55 \%$ \\
\hline 3 & 0.5482 & $13.67 \%$ & 44.27 & 0.158093182 & 7.859396212 & $31.41 \%$ \\
\hline 4 & 0.5000 & $12.47 \%$ & 56.74 & 0.11114578 & 5.52546742 & $36.93 \%$ \\
\hline 5 & 0.5000 & $12.47 \%$ & 69.21 & 0.111111111 & 5.523743877 & $42.45 \%$ \\
\hline 6 & 0.4518 & $11.27 \%$ & 80.47 & 0.072396871 & 3.599115962 & $46.05 \%$ \\
\hline 7 & 0.4163 & $10.38 \%$ & 90.85 & 0.049143796 & 2.443119673 & $48.50 \%$ \\
\hline 8 & 0.3564 & $8.89 \%$ & 99.74 & 0.020115182 & 1 & $49.50 \%$ \\
\hline 9 & 0.0105 & $0.26 \%$ & 100.00 & 0.101999496 & 5.070771842 & $54.57 \%$ \\
\hline 10 & 0.0000 & 0.00 & 100.00 & 0.111111111 & 5.523743877 & $60.09 \%$ \\
\hline
\end{tabular}

\section{Appendix 1: Axes, Eigenvalues and Rates of Variance}

\begin{tabular}{|c|c|c|}
\hline Question & Response Option & Count \\
\hline \multirow{3}{*}{ Entry Qualifications } & Access & 64 \\
\cline { 2 - 3 } & A-Levels & 105 \\
\cline { 2 - 3 } & Graduate & 11 \\
\cline { 2 - 3 } & Mature A-Levels & 13 \\
\cline { 2 - 3 } & Other Academic qualifications & 14 \\
\cline { 2 - 3 } & Other Vocational qualifications & 28 \\
\hline \multirow{4}{*}{ Exit Awards } & First class honours & 30 \\
\cline { 2 - 3 } & Upper second class honours & 141 \\
\cline { 2 - 3 } & Lower second class honours & 24 \\
\cline { 2 - 3 } & Sub-honours awards & 5 \\
\cline { 2 - 3 } & No award & 35 \\
\cline { 2 - 3 } & &
\end{tabular}

Appendix 2: Active Questions and Response Options 


\begin{tabular}{|c|c|c|c|c|}
\hline & $\begin{array}{c}\text { Relative } \\
\text { Weight (\%) } \\
\end{array}$ & $\begin{array}{c}\text { Squared distance } \\
\text { to origin }\end{array}$ & Axis 1 & Axis 2 \\
\hline \multicolumn{5}{|l|}{ Entry Qualifications } \\
\hline Access & 13.617 & 2.67188 & 13.69 & 19.81 \\
\hline A-Levels & 22.340 & 1.23810 & 0.57 & 10.90 \\
\hline Graduate & 2.340 & 20.36360 & 12.80 & 10.73 \\
\hline Mature A-Levels & 2.766 & 17.07690 & 21.79 & 0.86 \\
\hline Other Academic qualifications & 2.979 & 15.78570 & 0.17 & 0.14 \\
\hline Other Vocational qualifications & 5.957 & 7.39286 & 1.00 & 7.58 \\
\hline TOTAL & 50.000 & - & 50.01 & 50.01 \\
\hline \multicolumn{5}{|l|}{ Exit Awards } \\
\hline First class honours & 6.383 & 6.83333 & 38.04 & 1.90 \\
\hline Upper second class honours & 30.000 & 0.66667 & 0.32 & 8.95 \\
\hline Lower second class honours & 5.106 & 8.79167 & 5.91 & 2.99 \\
\hline No award & 7.447 & 5.71429 & 5.73 & 36.15 \\
\hline TOTAL & 48.936 & - & 49.99 & 49.99 \\
\hline
\end{tabular}

NB: categories making above average contributions to the structure of the axes are highlighted in bold

Appendix 3: Contributions of Active Categories to Axes 1 and 2 


\begin{tabular}{|c|c|c|c|c|c|}
\hline \multirow[t]{2}{*}{ Level of Analysis } & \multirow[t]{2}{*}{ B (Std Error) } & \multirow[t]{2}{*}{ Significance } & \multicolumn{3}{|c|}{ 95\% Confidence Interval for Odds Ratio } \\
\hline & & & Lower CI & Odds Ratio & Upper CI \\
\hline \multicolumn{6}{|c|}{$\begin{array}{l}\text { Pass at Level } 4 \\
\mathrm{R}^{2}=.09 \text { (Cox \& Snell), .18 (Nagelkerke). Model } \mathrm{X}^{2}(4)=18.27, \mathrm{p}=.001\end{array}$} \\
\hline Constant $^{\mathrm{a}}$ & $-2.90(0.44)$ & .000 & . & .06 &. \\
\hline Gender (male) & $1.77(0.58)$ & $.002 * *$ & 1.88 & 5.84 & 18.15 \\
\hline SEC (lower groups) & $1.56(0.54)$ & $.004 * *$ & 1.66 & 4.78 & 13.77 \\
\hline \multicolumn{6}{|c|}{$\begin{array}{l}\text { Pass at Level } 5 \\
\mathrm{R}^{2}=.09 \text { (Cox \& Snell), .17 (Nagelkerke). Model } \mathrm{X}^{2}(4)=18.61, \mathrm{p}=.001\end{array}$} \\
\hline Constant $^{\mathrm{a}}$ & $-2.86(0.43)$ & .000 & . & 0.06 & . \\
\hline Gender (male) & $1.65(0.58)$ & $.004 * *$ & 1.69 & 5.22 & 16.11 \\
\hline SEC (lower groups) & $1.67(0.53)$ & $.002 * *$ & 1.88 & 5.32 & 15.06 \\
\hline \multicolumn{6}{|c|}{$\begin{array}{l}\text { Pass at Level } 6 \\
\mathrm{R}^{2}=.11 \text { (Cox \& Snell), .19 (Nagelkerke). Model } X^{2}(4)=21.64, \mathrm{p}<.001\end{array}$} \\
\hline Constant $^{\mathrm{a}}$ & $-2.72(0.41)$ & .000 & 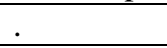 & 0.07 & . \\
\hline Gender (male) & $1.72(0.56)$ & $.002 * *$ & 1.85 & 5.58 & 16.82 \\
\hline SEC (lower groups) & $1.74(0.51)$ & $.001 * * *$ & 2.11 & 5.71 & 15.47 \\
\hline \multicolumn{6}{|c|}{$\begin{array}{l}\text { ‘Good’ Degree } \\
\mathrm{R}^{2}=.08 \text { (Cox \& Snell), .12 (Nagelkerke). Model X }{ }^{2}(3)=15.63, \mathrm{p}=.001\end{array}$} \\
\hline Constant $^{\mathrm{a}}$ & $-1.65(0.28)$ & .000 & 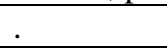 & 0.19 & . \\
\hline Gender (male) & $1.30(0.51)$ & $.011^{*}$ & 1.34 & 3.67 & 10.04 \\
\hline SEC (lower groups) & $1.22(0.41)$ & $.003^{* *}$ & 1.53 & 3.39 & 7.50 \\
\hline
\end{tabular}

a: constant or baseline model in which all predictor variables are omitted and it is assumed that all cases fall into the outcome category with the highest frequency (i.e. 'yes' in all levels of analysis in Table 5); * $\mathrm{p}<.05$; ** $\mathrm{p}<.005 ;{ }^{* * *} \mathrm{p}=.001$

Table 1: Significant predictors of progression 


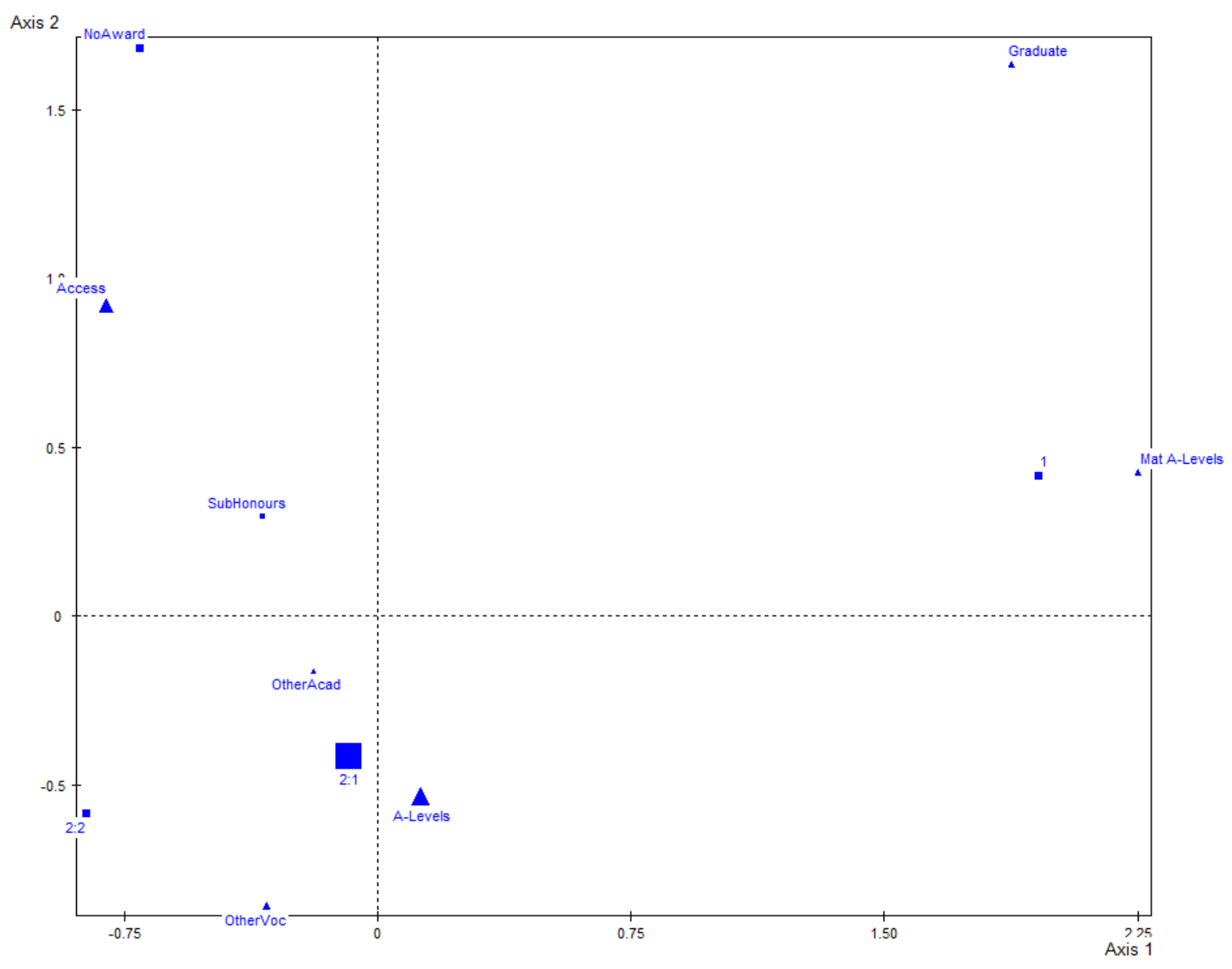

Figure 1: Cloud of active categories (Entry Qualifications \& Exit Awards) - Axes 1 and 2 $\triangle$ Entry Qualifications; $\square$ Exit Awards 


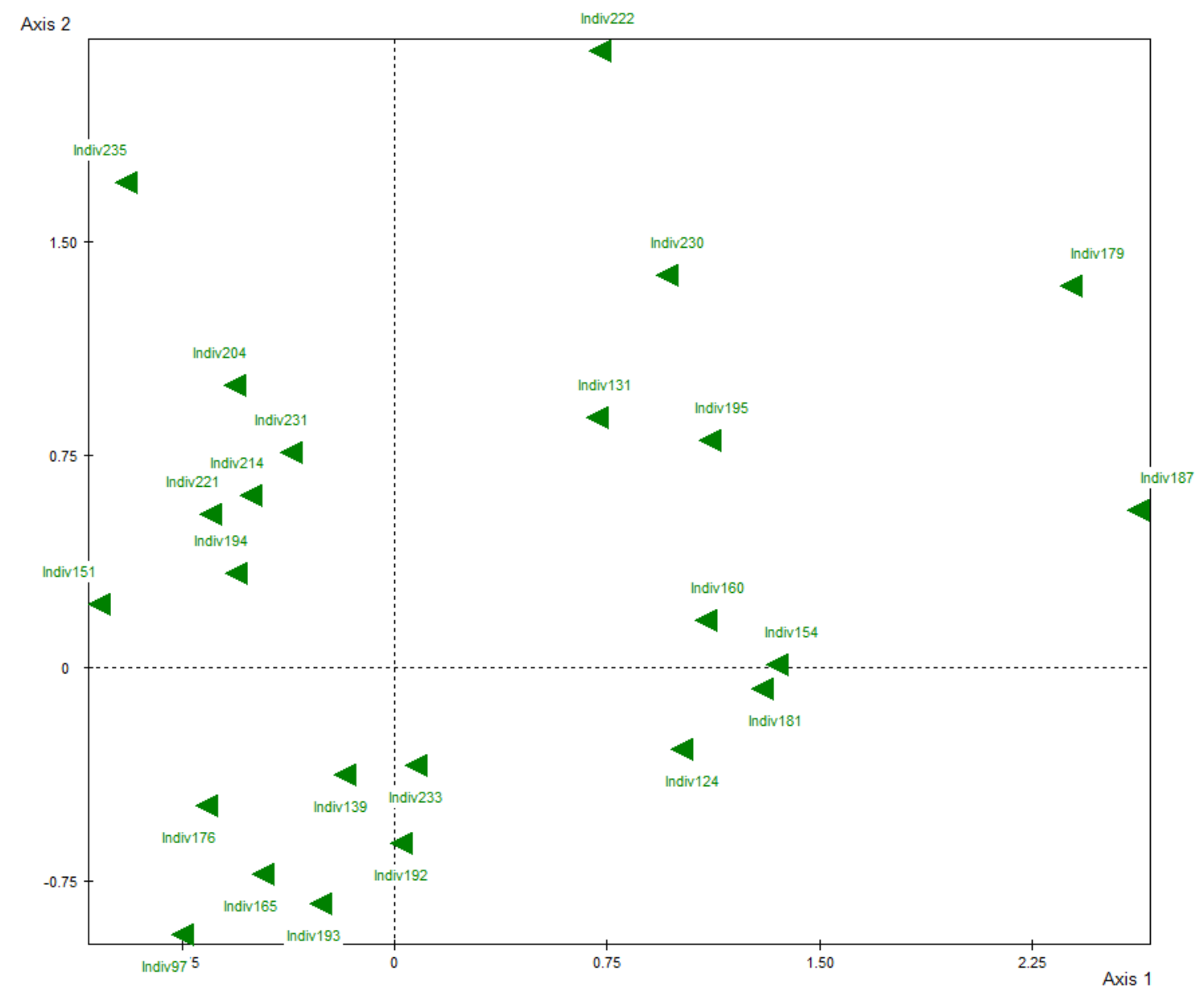

Figure 2: Cloud of Individuals - Axes 1 and 2 


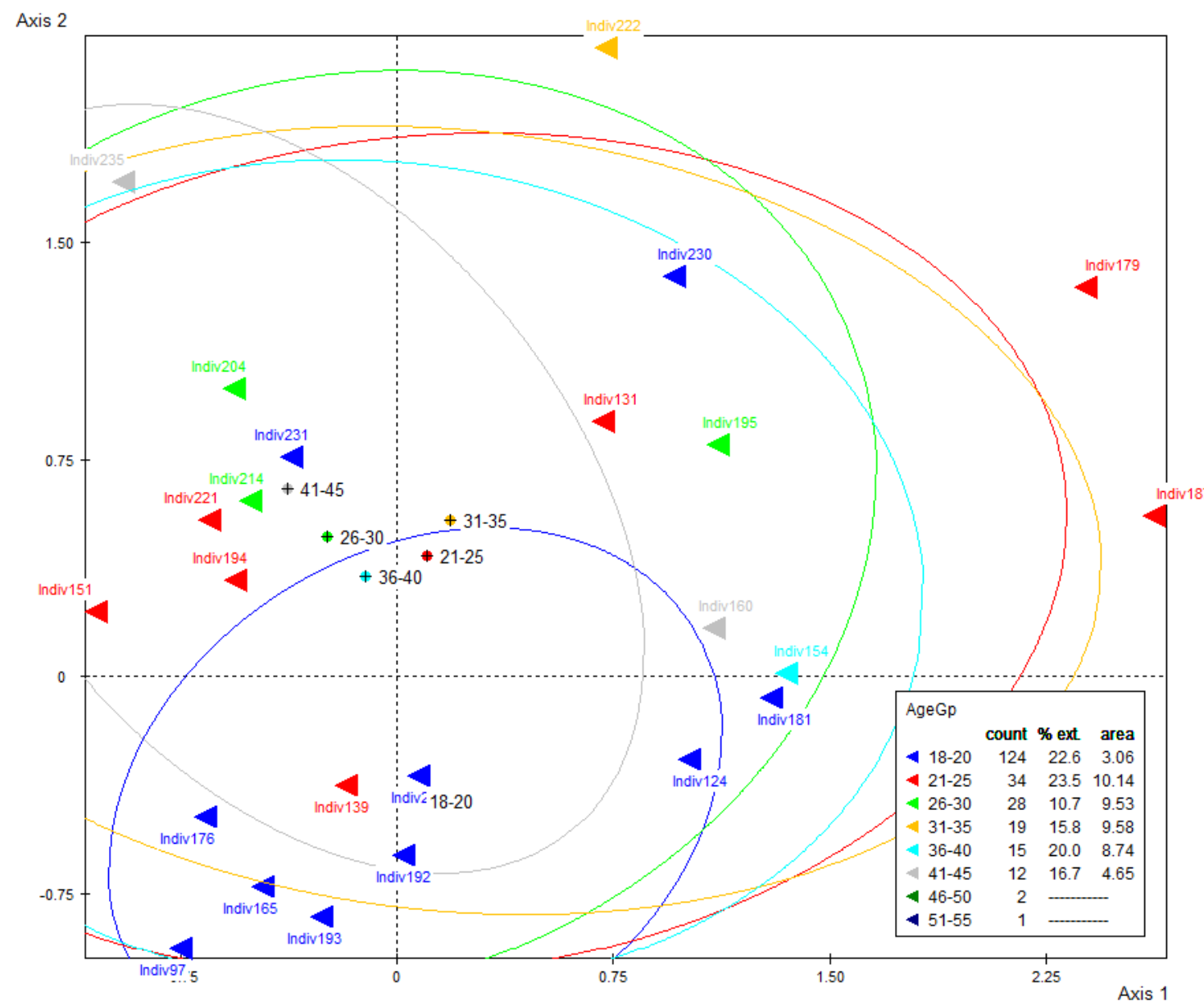

Figure 3: Cloud of individuals with concentration ellipses for Age at Entry - Axes 1 and 2 


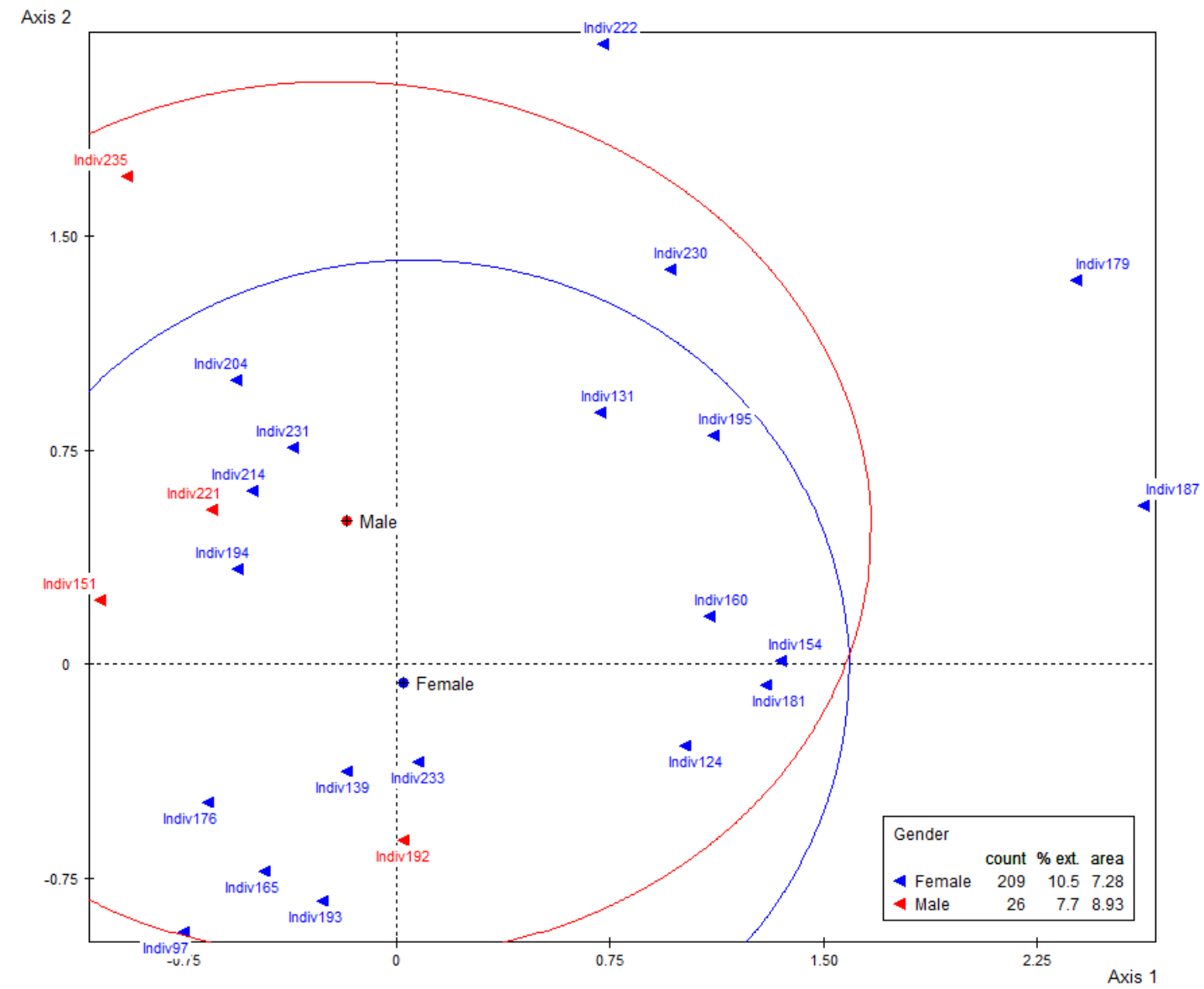

Figure 4: Cloud of individuals with concentration ellipses for Gender - Axes 1 and 2 


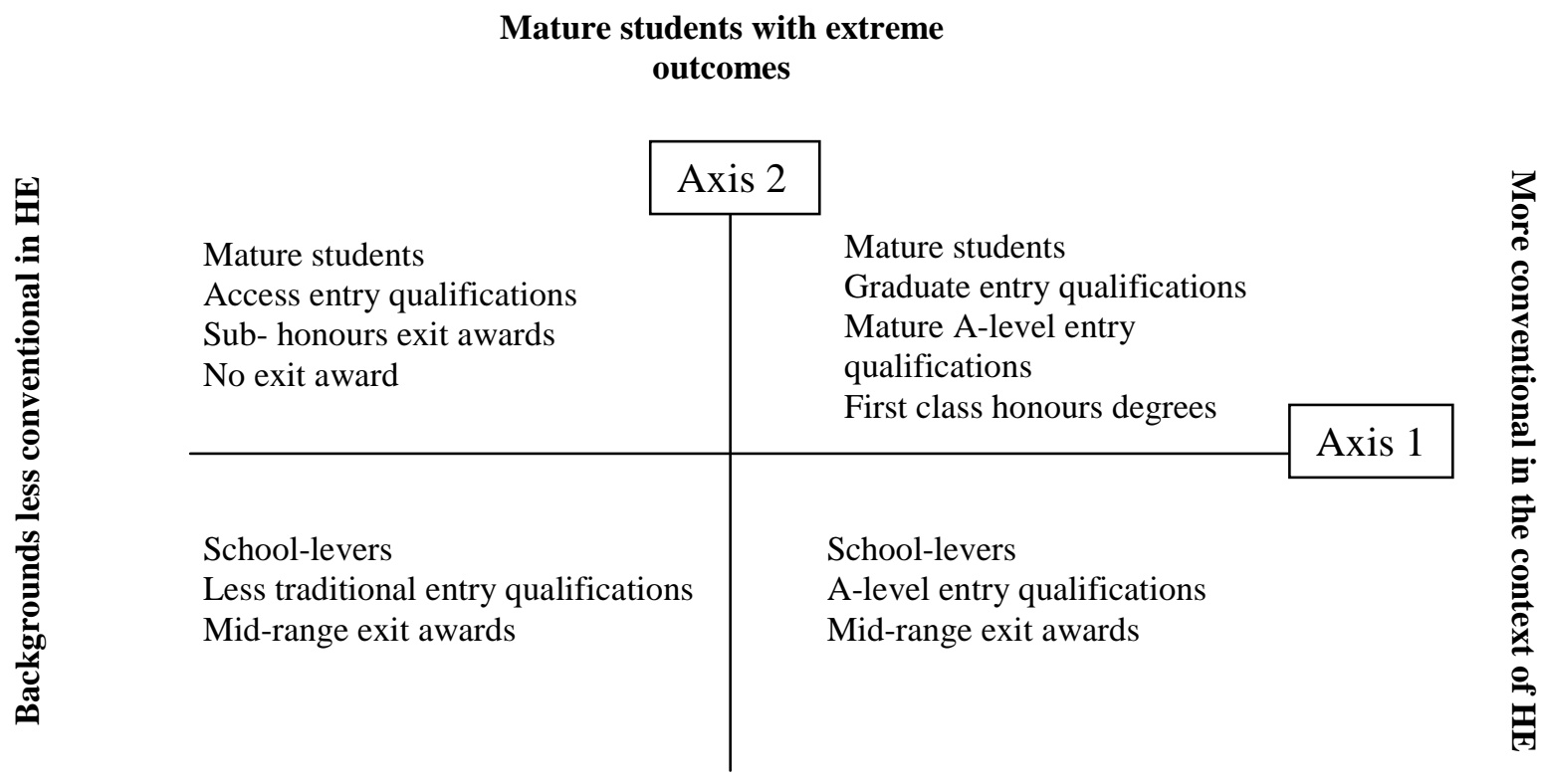

School-leavers with moderate

outcomes

Figure 5: Summarising the MCA of the progression routes data 